\title{
Synchronous Breast Cancer and Renal Leiomyoma
}

\author{
Caner Ediz ${ }^{1}$, Neslihan Kaya² and Serkan Akan ${ }^{1}$ \\ ${ }^{1}$ Department of Urology, Sultan Abdulhamid Han Education and Research Hospital, Istanbul, Turkey \\ ${ }^{2}$ Department of Pathology, Sultan Abdulhamid Han Education and Research Hospital, Istanbul, Turkey
}

\begin{abstract}
We present a case of a 49-year Turkish woman who had synchronous renal leiomyoma and breast cancer. The patient was evaluated for a suspicious breast mass; and renal mass was detected incidentally by contrast-enhanced computed tomography (CT). Diagnostic tru-cut biopsy was performed for both masses. Breast mass biopsy was reported as invasive ductal carcinoma (IDC) while renal biopsy was described as benign mesenchymal tumour. According to the biopsy results, the renal mass was followed for six months. For breast cancer, segmental mastectomy and concomitant sentinel lymph node biopsy was performed. Histologically, the tumour was reported as IDC and low-grade cribriform ductal carcinoma in situ (less than 1\%). Six months later, renal mass was excised by laparoscopic approach. Histopathological examination was consistent with renal leiomyoma. For both tumours, no recurrence within one year was found on follow-up.
\end{abstract}

Key Words: Renal leiomyoma, Breast cancer, Laparoscopy.

How to cite this article: Ediz C, Kaya N, Akan S. Synchronous Breast Cancer and Renal Leiomyoma. J Coll Physicians Surg Pak 2021; 31(10):1234-1236.

\section{INTRODUCTION}

Leiomyomasare benigntumours, and kidney is a very rarelocation for these tumours. Upto 45 cases of renal leiomyoma have been reported till date in English language medical literature. Synchronous renal leiomyoma and breast cancer was reported only in one case in the studies till date. ${ }^{1}$ This case report is to the best of our knowledge, the first case in the literature with simultaneous occurrence of renal leiomyoma ( $\mathrm{RL}$ ) and breast cancer in the same patient.

\section{CASE REPORT}

A 49-year woman in menopause was admitted with suspicious right breast mass in November 2018. In the physical examination, a solitary nodular mass was found in the upper outer quadrant of the right breast. No pathological lymph nodes were detected in the axillary area. It was reported as a low-density nodular opacity on mammography. Magnetic resonance imaging (MRI) was performed to evaluate nodular opacity in the breast tissue. The mass size was $9 \times 6 \mathrm{~mm}$ and location of mass was $8 \mathrm{~cm}$ far from the nipple. Breast mass was irregularly shaped with an irregular contour.

Correspondence to: Dr. Caner Ediz, Department of Urology, Sultan Abdulhamid Han Education and Research Hospital, Istanbul, Turkey

E-mail: drserkanakan@hotmail.com

Received: November 19, 2019; Revised: January 27, 2020; Accepted: March 13, 2020

DOI: https://doi.org/10.29271/jcpsp.2021.10.1234

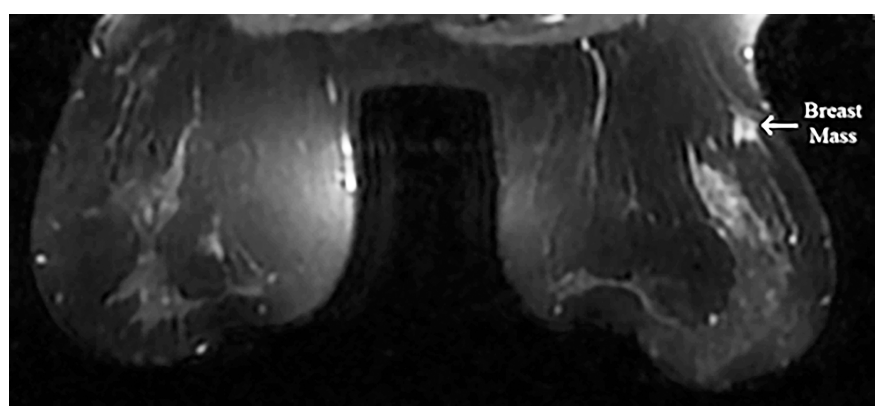

Figure 1: On the MRI, breast mass was hypointense in T1A, hyperintense appearance in $2 \mathrm{~W}$ and compatible with malignancy.

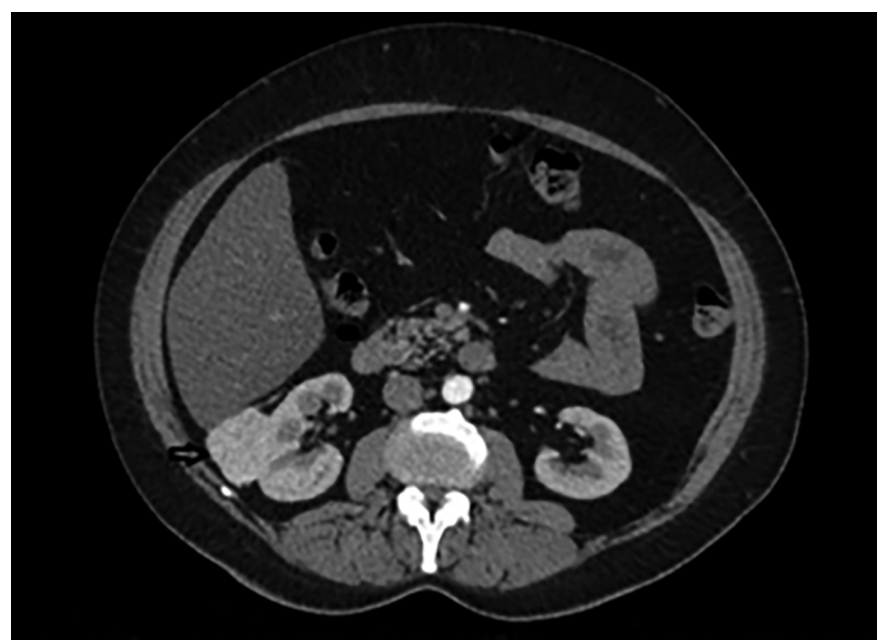

Figure 2: In abdominal contrast-enhanced computed tomography, approximately $53 \times 43 \times 35 \mathrm{~mm}$ hypervascular solid mass lesion.

On MRI, hypointense appearance in T1-weighted images and hyperintense appearance in T2-weighted images were reported in the right breast and these findings were compatible with malignancy (Figure 1). Left breast tissue was normal. Tru- 
cut biopsy was performed to confirm the diagnosis for suspicious breast mass. Pathology result was reported as infiltrating ductal carcinoma (IDC). To assess metastases, contrast-enhanced CT was planned and an approximately $53 \times 43 \times 35 \mathrm{~mm}$ hypervascular solid mass lesion in the right kidney in the posteroinferior region of Morison's pouch was found incidentally (Figure 2). Tru-cut biopsy was performed because the imaging of renal mass was not compatible with the diagnosis of either metastasis or primary renal cell cancer. Pathology result of renal mass was reported as a benign mesenchymal tumour. Therefore, segmental mastectomy and sentinel lymph node biopsy was performed for breast cancer. Breast mass was reported as IDC and low-grade cribriform ductal carcinoma in situ (DCIS) (less than $1 \%$ ) that was $0.9 \times 0.9 \times 0.8 \mathrm{~mm}$ with $\mathrm{pT} 1 \mathrm{~b}$ stage (Figure 3 ). While vascular invasion was not detected, perineural invasion was present. No tumour was observed at surgical margins. Hormone receptors were reported as negative. Sentinel lymph node biopsy was negative for tumor. After this surgical removal, adjuvant radiotherapy (RT) was performed due to breast conserving surgery (BCS) plus DCIS that posed a risk of local recurrence. Six months after BCS and RT, abdominal contrast-enhanced CT was repeated for evaluation of renal mass. The size of renal mass did not change in six months and excision of renal mass was planned. Laparoscopic nephron-sparing surgery was performed (Figure 4). Patient was discharged on the third postoperative day. In histopathological examination, a benign encapsulated leiomyomatous tumour composed of proliferation of benign smooth muscle cells was reported. There was whorled (fascicular) pattern of smooth muscle bundles separated by well vascularised connective tissue. No adipose tissue was seen. Immunohistochemistry confirmed myoid differentiation, with positivity for smooth muscle actin (SMA), muscle-specific actin (MSA), desmin, and hcaldesmon. Tumour cells did not show positive reaction for HMB-45, S-100 protein, estrogen receptors (ER), or progesterone receptors $(\mathrm{PR})$. Surgical margins were negative (Figure 5). The patient was followed regularly. At first year of follow-up, there was no recurrence of RL or IDC.

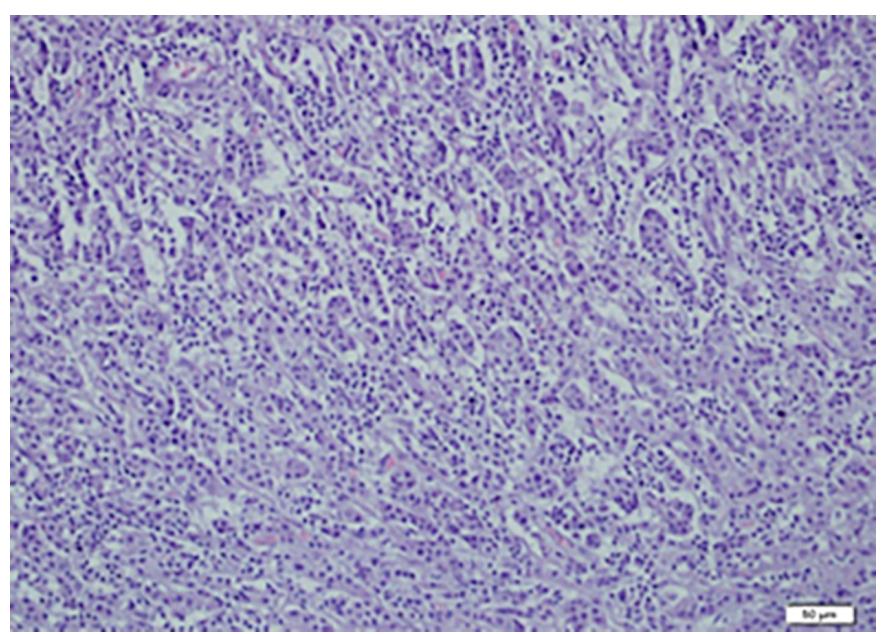

Figure 3: Microscopic examination of invasive ductal carsinoma. Tubular formations are prominent and tumorcells are pleomorphic.

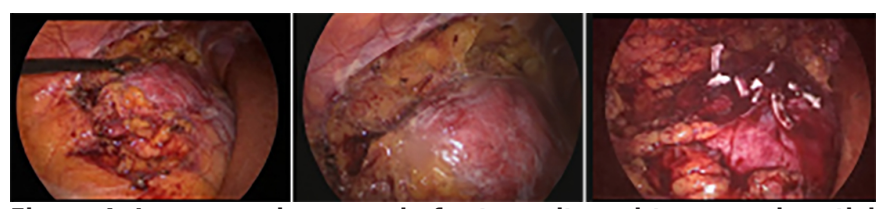

Figure 4: Laparoscopic removal of retroperitoneal tumor and partial nephrectomy.

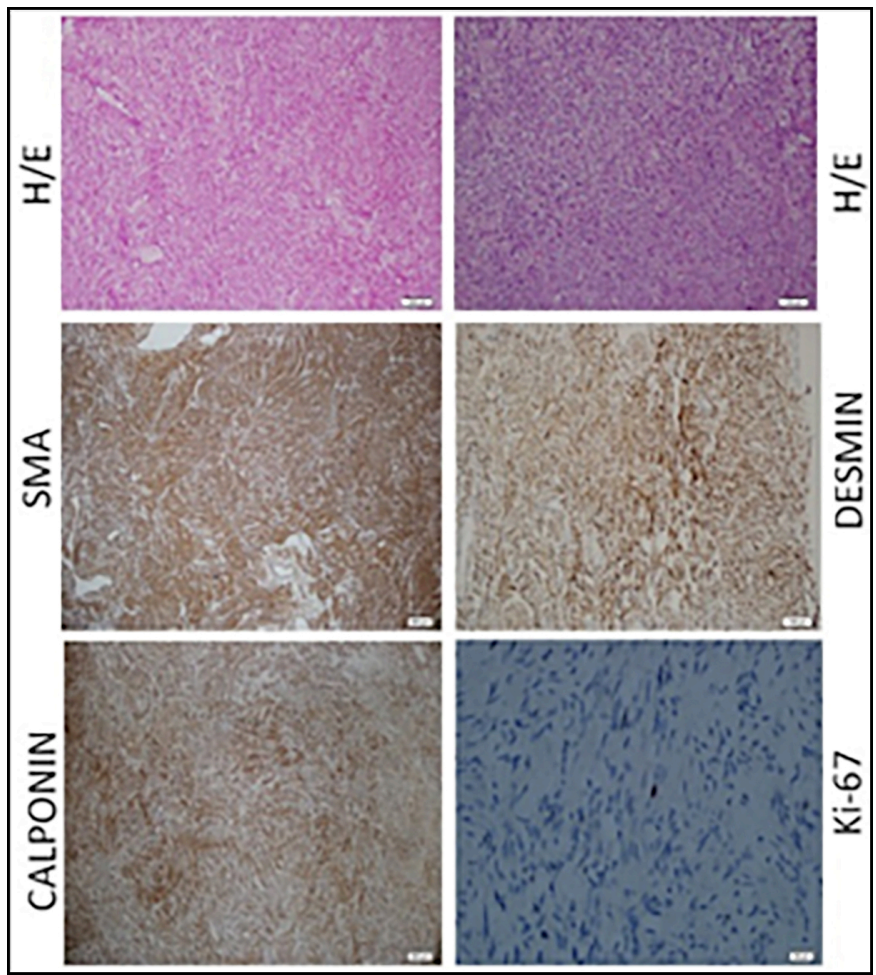

Figure 5: Typical histology of leiomyoma. Representative slides demonstrating the typical hematoxylin/eosin (H/E) slide and immunohistochemistry stains for smooth muscle antigen (SMA), desmin, calponin and Ki-67 in leiomyoma. Note the expanded leiomyoma tissue and increased nuclear size in leiomyoma smooth muscle cells. Immunoreactive SMA and calponin (brown stain) are localised to the cytoplasma of leiomyoma smooth muscle cells. Desmin shows nuclear and cytoplasmic staining. Prolipherative index is low with Ki-67 staining.

\section{DISCUSSION}

$\mathrm{RL}$ is a rare benign tumour that can originate in the retroperitoneal area including kidney. It is usually a single and unilateral tumour but Goren et al. have reported bilateral RLs in a 50-year woman. ${ }^{2}$ The etiology of RLs is unknown. Current findings point to cytogenetic and molecular genetic factors that include fusion of the genes EWSR1 and PBX3. ${ }^{3}$ But in this case report, no genetic factors were found on analysis. RLs include smooth muscle tissue and are similar to uterine leiomyomas.

Clinically, asymptomatic RLs are commonly detected in recent years due to widespread use of ultrasonography and computed tomography. They are usually non-metastatic and have no aggressive behaviour. ${ }^{4}$ In our case too, there were no symptoms and the tumor was managed conservatively due to concurrent breast mass. Size of RL did not change in six months. No metastasis was found. 
Association of retroperitoneal leiomyoma and breast cancer has been reported in only one case in the literature. ${ }^{1}$ However, this case was not a synchronous tumour, but it was a metachronous tumour. Four years before diagnosis of retroperitoneal leiomyoma, the patient received an anti-estrogen therapy for breast cancer. In another case, Victory et al. have reported that the gonadotrophic hormones seem to influence the development of this complication. ${ }^{5}$ But there is not enough supporting evidence about this unexplored issue. In our case, we are reporting for the first time the synchronous occurrence of $R L$ and breast cancer in the same patient. This coexistence does not support its association with anti-estrogen therapy. Also in this case, there was no positive reaction for ER and PR in tumourcells. It may have been caused by another genetic factor that is not described or some environmental factors.

In the literature, Gupta et al. have published the largest series on smooth muscle neoplasms and have reported that RLs uniformly expressed all smooth muscle markers and were ER/PR/WT1 positive in nearly all cases. ${ }^{6}$ But in our case, tumour cells did not show positive reaction for ER and PR.

EAU guidelines recommend partial nephrectomy (PN) for patients with T1 tumours. Laparoscopic nephron-sparing surgery is a safe technique for renal masses that belong to T1 stage. Therefore, we performed laparoscopic PN in this case. The renal mass was easily dissected from the posterior surface to the lateral area of the kidney. Renal ischemia was not experienced during PN as opposed to routine practice. Total operation time was 90 minutes and intraoperative bleeding was about $50 \mathrm{ml}$. We do not recommend routine renal ischemia in small asymptomatic RLs, because the tumor does not exhibit aggressive behaviour and the tendency for invasion is poor. RLs generally do not recur after complete excision.

$\mathrm{RL}$ is a rare benign mesenchymal tumor of the kidney with favourable prognosis. In addition to the possibility of metastasis to kidney, in cases of incidental detection of renal masses, RL should be kept in mind in patients with breast cancer who undergo metastatic screening. Nephron-sparing surgery with zero ischemia is a possible and feasible technique in patients with small RLs (pT1).

\section{PATIENT'S CONSENT:}

Written informed consent was obtained from the patient for his anonymised information to be published in this article.

\section{CONFLICT OF INTEREST:}

The authors declared no conflict of interest.

\section{AUTHORS' CONTRIBUTION:}

EC: Contributed to the conception and design of the case report,

EC, AS: Collection of data.

$\mathrm{EC}, \mathrm{KN}$ : Revision the manuscript.

EC, AS, NK: Prepared figures and performed last evaluation.

All authors read and approved the final manuscript.

\section{ACKNOWLEDGEMENT:}

The authors would like to thank the entire staff of the Department of Urology and Pathology in Sultan Abdulhamid Han Education and Research Hospital.

\section{REFERENCES}

1. Moon JJ, Mais DD. Retroperitoneal lipo-leiomyomata in a woman with history of breast cancer and antiestrogen therapy. American Society Clin Oncol 2017; 35(15).

2. Goren MR, Erbay G, Ozer C, Goren V, Bal N. Bilateral renal leiomyoma with 5-year follow-up: Case report. Can Urol Assoc J 2015; 9(9-10):E734-6. doi: 10.5489/cuaj.3011.

3. Panagopoulos I, Gorunova L, Bjerkehagen B, Heim S. Fusion of the genes EWSR1 and $P B X 3$ in retroperitoneal leiomyoma with $\mathrm{t}(9 ; 22)$ (q33;q12). PLoS One 2015; 10(4): e0124288. doi: 10.1371/journal.pone.0124288.

4. Brunocilla E, Pultrone CV, Schiavina R, Vagnoni V, Caprara G, Martorana G. Renal leiomyoma: Case report and literature review. Can Urol Assoc J 2012; 6(2):E87-90. doi: 10.5489/cuaj.11159.

5. Victory R, Romano W, Bennett J, Diamond M. Clinical gynecology. London: Churcill living stone an imprint of Elsivier Inc 2006; 179-202.

6. Gupta S, Jimenez RE, Folpe AL, Cheville JC. Renal leiomyoma and leiomyosarcoma: A study of 57 cases. Am J Surg Pathol 2016; 40(11):1557-63. doi: 10.1097/PAS. 0000000000000681. 